\title{
LA PERSPECTIVA DE GÉNERO
}

\author{
GENDERS PERSPECTIVE
}

\section{Ignasi Brunet Icart}

Universidad Rovira i Virgili, Tarragona. España/Spain ignasi.brunet@urv.cat

Recibido/Received: 18/02/08

Aceptado/Accepted: 18/06/08

\section{RESUMEN}

Este artículo tiene por objetivo exponer cómo los procesos de pobreza y exclusión social de las mujeres y de los hombres hay que analizarlos desde la perspectiva de género. Perspectiva que permite explicar de qué forma la posición económica de las mujeres viene determinada por la división sexual del trabajo, y se explica gracias a la existencia del orden patriarcal, al sistema de dominación masculina en el que hombres y mujeres son definidos como seres humanos diferentes, cada uno de ellos con sus propias oportunidades, roles y responsabilidades.

\section{PALABRAS CLAVE}

Género, patriarcado, sexualidad, pobreza.

\begin{abstract}
This article takes as a target to exhibit how the processes of poverty and of social exclusion of the women and of the men it is necessary to analyze from the perspective of genre. Perspective that allows to explain how the economic position of the women comes determined by the sexual division of the work, and that is explained thanks to the existence of the patriarchal order, the system of masculine domination in which men and women are defined as human different beings, each of them with their own opportunities, rolls and responsibilities.
\end{abstract}

\section{KEYWORDS}

Gender, patriarchy, sexuality, poverty.

\section{INTRODUCCIÓN}

Las diversas medidas históricas de la pobreza han pasado por encima de los procesos de pobreza y de exclusión social de las mujeres y de los hombres hay que analizarlos de forma diferenciada, pero no de forma separada, perdiendo así la 
observación más general sobre el modo en que el género es construido en y funcionalmente para el sistema capitalista (Tortosa, 1993, 2001; Boserup, 1993; Kabeer, 1997, 1998). La variable género ha permitido al feminismo desarrollar un análisis explicativo-diagnóstico de la situación de las mujeres a través de la historia, la cultura y las sociedades. El feminismo, como teoría crítica que se inserta en la tradición de las teorías críticas de la sociedad, constituye, por otro lado, "un referente necesario si no se quiere tener una visión distorsionada del mundo ni una autoconciencia sesgada de nuestra especie" (Amorós y De Miguel, 2007c:21). Una teoría que es el marco de un movimiento social y político cuyo objetivo es el reconocimiento de los derechos de las mujeres como parte inalienable, integral e indivisible de los derechos humanos universales (Folguera, 2006a), y que exige "la autodesignación de las propias mujeres en el plano teórico y la autodesignación de espacios que le es correlativa en el práctico" (Amorós, 2006:226). Exigencia que tendrá importantes consecuencias al poner de manifiesto el carácter artificial de "lo femenino", y el descubrimiento de la realidad del patriarcado. La elaboración de este constructo discursivo va a explicar la sistemáticamente fraudulenta usurpación de lo universal por parte de una particularidad, la constituida por el conjunto de quienes detentan el poder. Usurpación que Friedan (1974) denunció en "La mística de la feminidad", expresión que alude "a una concepción esencialista de la feminidad según la cual las mujeres tendrían una naturaleza especial y consustancial que sólo se puede desarrollar plenamente en la pasividad sexual, en el sometimiento al varón y en consagrarse amorosamente a la crianza de los hijos" (Perona, 2007:21).

La reflexión antipatriarcal del feminismo va a poner de manifiesto de forma consciente que las bases mismas de la vindicación femenina se encuentran en el solapamiento de lo masculino con lo genéricamente humano. El planteamiento mismo de la vindicación está en que la impostación de la universalidad produce discriminación, y si el feminismo lo ha denunciado, es porque en la base misma de su formulación había un vicio de origen: la adscripción de las mujeres a la esfera privada -doméstica-. Esta adscripción es el mecanismo por el que se consuma la exclusión de las mujeres de las promesas ilustradas de igualdad y libertad. Fuera de lo público no había ni razón, ni ciudadanía, ni igualdad, ni legalidad, ni reconocimiento de los otros (Pateman, 1995). De este modo, en la modernidad "las dos esferas se constituyen con lógicas y simbólicas contrapuestas $\mathrm{y}$, frente a una supuesta complementariedad de identidades y funciones, aparecen rígidamente separadas y jerarquizadas. El discurso teórico de la modernidad y las nuevas producciones científicas se encargarán de legitimar este orden social a través de la ideología de la naturaleza diferente y complementaria de los sexos que se convirtió, tanto desde la filosofía como desde las nuevas ciencias sociales, en la ideología legitimadora de estos dos espacios e identidades" (Amorós y De Miguel, 2007c:65). 
En este artículo se expone la perspectiva de género desarrollada por el pensamiento feminista; perspectiva que hay que tener en cuenta para el análisis de la pobreza y la exclusión social. En el contexto de la generización de los conceptos de pobreza y exclusión social, se destacan dos aproximaciones: La primera, orienta su estudio a la feminización de la pobreza, que hizo fortuna a finales de la década de los 90 y que sigue utilizándose en la actualidad en investigaciones de ámbito internacional (López Larrea, 1989; Allo y Harcourt, 1997; PNUD, 1995; Andersen y Larsen, 1998; Marcoux, 1998; Martínez, 2001). Se trata de una perspectiva que defiende que las mujeres son más pobres que los hombres, y el concepto de feminización se utiliza para indicar que la pobreza tiene una mayor incidencia en la mujer que en el hombre, o que la pobreza de las mujeres es más severa que la de los hombres, o que la incidencia sobre las mujeres está creciendo en comparación con los hombres. Pero, la expresión feminización de la pobreza no deja de ser una fórmula vacía ya que se la formula como si tuviera un contenido suficiente y reconocido, y únicamente señala que la proporción de mujeres sobre el total de pobres ha aumentado; la segunda, orienta su estudio a la relación entre el proceso de empobrecimiento y exclusión y el género. Se trata de ver que las mujeres son pobres en tanto que esta situación está condicionada, entre otras variables, por el género, en otras palabras, la experiencia de la pobreza y exclusión está condicionada por las identidades de género. Esto significa que hombres y mujeres son definidos como seres humanos diferentes, cada uno de ellos con sus propias oportunidades, roles y responsabilidades. Y ello tiene que ver con la división del trabajo, que es fundamentalmente división sexual, observable no únicamente en el vínculo entre la feminización de la fuerza de trabajo y el empeoramiento de las condiciones de trabajo (Benería, 1992), sino también en el contenido y las condiciones de trabajo de hoy (Maruani, 2002, 2007; Maruani et al., 2000; Carrasco et al., 2003, 2004; Castaño et al., 1999).

\section{GÉNERO Y PATRIARCADO}

Con la constitución del término gramatical género como categoría analítica, el feminismo logró un lugar de legitimidad académica (Posada, 1998, 2002; Lauretis, 2000; Benhabib, 1990). Una categoría que surgió en un momento de gran turbulencia epistemológica y que supuso "un debate en el que unos afirman la transparencia de los hechos y otros insisten en que la realidad es construida. En el espacio abierto por este debate y desde el lado de la crítica de la ciencia, desarrollada por las humanidades, así como de la crítica al empirismo y al humanismo hecha por los post-estructuralistas, las feministas han comenzado a tener no sólo voz propia sino también aliados académicos y políticos" (Scott, 1984:32) en la tematización del patriarcado como matriz que configura la identidad, así como la inserción en lo real de hombres y mujeres. Específicamente, mediante esta categoría el feminismo se percató que aunque el capitalismo necesita 
a "alguien" que se quede en la esfera privada y compre todo lo que el mercado ofrece para esta esfera, no necesita que ese "alguien" sea precisamente las mujeres; quién sea ese "alguien" es algo que la estructura del capitalismo deja indeterminado. El que esa variable se llene con las mujeres es algo que sólo se explica gracias a la existencia del orden patriarcal (Perona, 2007). Un orden que, según Jónasdóttir (1993), se sostiene hoy fundamentalmente por "relaciones sexuales libres" (no por las forzadas o por la violencia sexual) que se establecen entre hombres y mujeres. En su tesis política "El poder del amor. ¿Le interesa el sexo a la democracia?", Jónasdóttir (1993) argumenta que en la actualidad el patriarcado se sostiene en las condiciones políticas del amor, y define el amor como prácticas de relación sociosexuales, y no sólo como emociones que habitan dentro de las personas. Su tesis es sobre el amor como poder humano, materialista, alienable, y como práctica social, básico para la reproducción del patriarcado, y que afecta a las condiciones de trabajo (productivo y reproductivo) de las mujeres; condiciones que no son el resultado de un determinismo económico, sino el fruto de la conjunción entre los requerimientos del sistema productivo y la oferta del sistema reproductivo.

Para Jónasdóttir (1993) las relaciones de subordinación / dominio entre hombre y mujeres se sitúan, por mediación de la práctica del amor, en la esfera reproductiva, en el plano de la familia, y se proyectan, después, sobre las relaciones sociales fuera de la familia. Por ello no es posible apreciar el carácter del patriarcado moderno sin el significado de la separación entre la familia - lo privado- y lo público; separación que supuso la privatización y psicologización de la función materna mientras excluía a las madres del trabajo remunerado y, por extensión, del status de ciudadano, dado que la reclusión de las mujeres al plano de lo privado supuso que se les privase de una serie de derechos consagrados como universales (Puleo, 2007; Millet, 1995; Greer, 1985, 2000). Privación en el sentido de que el concepto de ciudadanía ha sido definido desde la óptica masculina, y no contempla la realidad específica de las mujeres como sujetos de diferentes derechos que los hombres, ya que "cuando a las mujeres se les niega la democracia y los derechos humanos en la esfera privada, sus derechos humanos en la esfera pública también se ven afectados" (Folguera, 2006a:88).

La exclusión de las mujeres del trabajo remunerado las recluyó al ámbito privado-familiar y, así, durante los siglos XIX y XX se conformó y consolidó el modelo de mujer "ama de casa" y "madre amantísima" que realiza su actividad cotidiana en el ámbito de lo privado-familiar y coopera con un esposo que trabaja fuera del hogar, siendo ambos mantenedores del grupo familiar. Hasta fechas muy recientes, subraya Brullet (2004), ser una buena madre y esposa significaba atender a los hijos en sus necesidades físicas, psicológicas, emocionales y morales, marcar pautas de vida cotidiana, realizar el seguimiento escolar, atender el hogar, al marido y estar disponibles para cubrir las necesidades del grupo doméstico las veinticuatro horas del día. Ser padre significaba traer el dinero a casa y marcar la 
ley dentro del hogar, aunque en la actualidad esta división sexual del trabajo se ha modificado a partir de la incorporación masiva de las mujeres al mercado de trabajo. Incorporación que ha impuesto la "doble presencia" de las mujeres (trabajo remunerado y trabajo doméstico-familiar) (Picchio, 1992, 1999). Una "doble presencia" condicionada por los modelos de conductas sociales considerados adecuados para cada género, que dan cuenta de la segregación de los empleos por género. Segregación que es el mecanismo primario que "en la sociedad capitalista mantiene la superioridad de los hombres sobre las mujeres, porque impone salarios bajos para las mujeres en el mercado de trabajo. Los salarios más bajos mantienen a las mujeres dependientes de los hombres porque las impulsa a casarse. Las mujeres casadas deben realizar trabajos domésticos para sus maridos, de modo que son los hombres los que disfrutan tanto de salarios más altos como de la división doméstica del trabajo. Esta división doméstica del trabajo, a su vez, actúa debilitando la posición de las mujeres en el mercado de trabajo. Así, el mercado de trabajo perpetúa la división doméstica del trabajo y viceversa" (Hartmann, 1994:258). Y es que las mujeres llevamos "al ámbito del trabajo las connotaciones simbólicas del lugar que ocupamos en la estructura de la familia y, a su vez, se traducen en nuestra situación en la familia los efectos de la posición devaluada en que nos encontramos en el ámbito del trabajo" (Amorós, 2006:377).

Este hecho explica que para Hartmann (1981) al patriarcado se le pueda reconocer en las relaciones que los hombres mantienen con las mujeres dentro del sistema capitalista; relaciones entre hombres y mujeres que no son casos aislados o asuntos privados de pareja, sino que son relaciones sistémicas (Amorós y De Miguel, 2007c) que se sostienen en la separación entre lo público y lo privado, y que tienen "un origen liberal con la necesidad de la afirmación de la familia burguesa, que se perfila frente a la familia aristocrática del Antiguo Régimen y debe diferenciarse asimismo de la incipiente clase obrera. Se crea la ideología -y la realidad- de las esferas separadas, los hombres para lo público y las mujeres para lo doméstico y la crianza de la prole. El feminismo no sólo revela esta separación de esferas sino la dialéctica entre ambas. Para que pueda existir, ese dominio público de los derechos tiene que descansar sobre la negación de los mismos para las mujeres; el mundo de la producción necesita para sobrevivir de unos servicios para reproducirse, y éstos se hallan encomendados a las mujeres, reinas de la domesticidad" (Osborne, 2007:214).

El significado de la separación público/privado se vuelve claro, para Pateman (1995), cuando se pone en el contexto del contrato sexual, de la división sexual entre las esferas pública y privada. El perfil de género de las teorías del contrato social está en que éste ha sido construido a partir de la diferencia sexual, que marca la línea divisoria entre libertad -plano público- y subordinación -plano privado-. Pateman presenta el contrato social como un pacto patriarcal por el que los varones generan vida política a la vez que pactan mediante un contrato sexual los términos de su control sobre las mujeres. Contrato sexual por el que mediante la 
fraternidad, los varones se constituyen como "maridos", "trabajadores" y "ciudadanos", y relegan a la esfera privada, al espacio de la naturaleza, a las mujeres, y del que derivan de manera "natural" determinadas tareas, responsabilidades y comportamientos. Por consiguiente, es la división sexual del trabajo lo que explica las desigualdades y los problemas de acceso a la esfera pública de las mujeres en el mundo contemporáneo. De ahí que para Jónasdóttir (1993) es la utilidad del sexo femenino en el orden político moderno lo que constituye el argumento base del orden patriarcal actual, al ser la sexualidad una construcción política, un campo de poder independiente de las determinaciones socio-económicas, pero no por ello, menos real y material (Hartmann, 1981).

Jónasdóttir (1993) argumenta que la organización de la sexualidad en nuestras sociedades, en las que los hombres ejercen la autoridad que les da el "poder del amor" (explotando la necesidad que la mujer tiene de amar y ser amada) es el vector de opresión más importante en las mujeres de hoy, desplazando el trabajo y las determinaciones económicas del protagonismo que le otorga el marxismo (Molina, 2000, 2007; Hartmann, 1981, 1994; Amorós, 2006; Barry, 1987, 1994, 2007). En todo caso, la historia del contrato sexual de Pateman muestra cómo la diferencia sexual, como diferencia política, sustenta el patriarcado, y que Amorós (2005a, 2005b, 2006) define, siguiendo a Hartmann (1994), como un conjunto de pactos interclasistas entre varones, tales que les permiten tener bajo su control a las mujeres. En la medida y en el nivel en que estos pactos son operativamente eficaces, se afirma que el patriarcado existe. Existencia sustentada en la adjudicación diferencial de espacios, tareas, deseos, derechos, obligaciones y prestigio (Molina, 1994a, 1994b, 2000; Maquieira, 2006b).

El patriarcado, como conjunto de pactos interclasistas entre varones, es un orden genérico de poder (Lagarde, 1996), basado en un modo de dominación de los varones sobre las mujeres; un modo de dominación que "refuerza el control capitalista; y a su vez, los valores capitalistas, delimitan la definición de lo que es bueno para el patriarcado" (Hartmann, 1981, 27). El patriarcado, "como conjunto de relaciones sociales entre los hombres, que tiene una base material, y que a través de jerarquías, establece o crea interdependencias y solidaridad entre los hombres" (Hartmann, 1981:14), permite que los hombres mantengan su poder, controlando el "acceso de las mujeres a los recursos, controlando su fuerza de trabajo en lo doméstico y restringiendo su sexualidad. Con ello sacan un provecho en términos de servicios personales que les dispensa a ellos de realizar muchas tareas desagradables, dentro y fuera de casa (como limpiar los baños o servir cafés en las oficinas), o bien en términos de servicios sexuales. En el patriarcado, en fin, se trata de una apropiación por parte de los hombres de algo que tienen las mujeres o que hacen las mujeres, lo que las sitúa a ellas en unas relaciones de dominación, en un modo de producción opresor e injusto" (Molina, 2007:172). 


\section{SEXO Y DIFERENCIA DE LO FEMENINO}

Sobre la constatación obvia del dimorfismo sexual de la especie humana se ha construido, por una parte, el patriarcado o el sistema sexo-género que Benhabib (1990) define como la constitución simbólica y la interpretación socio-histórica de las diferencias anatómicas entre los sexos. El sistema de sexo-género es la red mediante la cual las sociedades y las culturas reproducen a los individuos incardinados, y los distintos sistemas de sexo-género históricamente conocidos han colaborado en la explotación y opresión de las mujeres. El patriarcado o sistema sexo-género otorga a los hombres el derecho de acceso sexual a los cuerpos de las mujeres y el derecho de mando sobre el uso de los cuerpos de las mujeres. Un derecho de acceso y mando apoyado en una construcción llamada sexo y que está tan culturalmente construida como el género (Lauretis, 1987, 2000). Como indica Butler (1990, 2001a, 2001b), el sexo es ya una categoría dotada de género, y éste es también el medio discursivo mediante el cual la "naturaleza sexuada" o "sexo natural" se establece y se produce como prediscursivo, previo a lo social, una superficie sobre la que actúa lo social. Para Barry (2007:201), ser sexualizada por lo social es verse "reducida al sexo corporal de una, o sea, las mujeres se reducen a través de la adscripción colectiva de conductas sexuales y de las características que las reducen a sus cuerpos, que las hacen inmanentes al sexo. La sexualización trata al sexo como si tuviera vida propia, una vida localizada sobre y dentro del cuerpo femenino". Con la sexualización, concluye Barry (2007:202), se confiere a las mujeres "una identidad colectiva (las mujeres son...) y se toman como un todo indiferenciado, un grupo biológico constituido, fundamentalmente, por funciones sexuales y reproductivas".

Las mujeres son objetivadas para la procreación de la especie y la constitución de la dictadura heterosexual. La heterosexualidad es una construcción social y cultural que exacerba las diferencias "naturales", biológicas y recrea así el género (Butler, 2001a, 2001b; Witting, 1981). Desde la perspectiva de Lauretis (2000), la construcción cultural del sexo como género y la asimetría que caracteriza en todas las culturas a los sistemas de sexo-género (aunque a cada uno de un modo particular) se entienden como sistemáticamente ligados a la organización de la desigualdad social (Oliva, 2007). Para Lauretis (2000:9), sí "las representaciones de género son posiciones sociales que llevan consigo diferentes significados, el que alguien sea representado y se represente a sí mismo como varón o mujer implica el que asume la totalidad de los efectos de este significado". Para Butler (2001a), afirmar que hay una mujer o un hombre natural o biológico que después se transforma en una mujer socialmente subordinada o en un hombre socialmente supraordenado, implica apoyarse en una concepción que entiende el sexo anterior a la ley en el sentido de que no está cultural ni políticamente determinado, proporcionando así la materia prima de lo social, por así decirlo, que empieza a tener significado sólo mediante su sometimiento a las reglas de parentesco y 
después de hacerlo. Sin embargo, la consideración del "sexo como materia", "sexo como instrumento de significación cultural" es una formación discursiva que funciona como un fundamento naturalizado para la distinción naturaleza/ cultura y para las estrategias de dominación que esa distinción apoya. Pero, también, implica caer en una lectura de lo natural como algo ajeno a lo histórico, a lo social.

Butler (2001a) define lo natural como un acontecer con lo social, con el hacer de las personas, y en el sentido de que no hay más naturaleza que la que acontece con las personas, los seres humanos, de ahí que el sexo tal vez siempre fue género, con la consecuencia de que la distinción entre sexo y género no existe como tal. De hecho, los diferentes actos del género crean la idea de género y sin estos actos, el género no existiría. En realidad, entender el sexo y la sexualidad como algo anterior a la ley es en sí la creación de la ley. Al respecto, Foucault (1984) mostró que estar sexuado significa estar sujeto a una serie de reglamentaciones sociales y mantener que la ley dirige estas reglamentaciones como formadoras del sexo, género, de los placeres y de los deseos. En el caso de Butler (1990), los actos los sesgos y los deseos producen el efecto de que existe una sustancia o núcleo interior, pero realmente actúan en la superficie del cuerpo, "mediante un juego de sugerencias significativas que nos hacen tomar la identidad como principio organizador y como causa. Tales actos, gestos y prácticas, generalmente construidos, son preformativos en el sentido de que la esencia de la identidad que parecen expresar se convierte en una fabricación manufacturada y sostenida mediante signos corporales y otros medios discursivos" (Oliva, 2007:31).

Por otra parte, sobre la constatación del dimorfismo sexual emerge la propuesta del feminismo cultural (Echols, 1983), y del feminismo de la diferencia que defiende la existencia de una cultura propia y específica para las mujeres, y que su explotación "está basada en la diferencia sexual y sólo por la diferencia sexual puede resolverse" (Irigaray, 1994:9). Es más, se afirma, señala Posada (2007a, 2007b), que el encuentro entre una mujer y un hombre puede alcanzar una dimensión de universalidad si tiene lugar en la fidelidad de cada uno a su género. Más específicamente, "las mujeres necesitan una cultura adaptada a su naturaleza" (Irigaray, 1994:69), dado que el ser humano es dos: lo masculino y lo femenino, el orden masculino y el orden femenino (Muraro, 1992, 1994). El orden femenino es el orden materno, que es el orden simbólico para las mujeres por excelencia y sólo en el reconocimiento de este orden es posible, para Muraro, la independencia femenina. Sólo eso "nos permite independizarnos del orden masculino y jugar desde otros parámetros simbólicos: desde el orden simbólico de la madre y de su autoridad. Sin aceptarlos, se produce según Muraro el desorden más grande que pone en duda la posibilidad misma de la libertad femenina" (Posada, 2001:311).

El feminismo cultural ecuaciona, entonces, la liberación de las mujeres con el desarrollo y la preservación de una contracultura femenina, abogando por un retorno a la familia tradicional como forma de proteger a las mujeres contra la violencia masculina (Jeffreys, 1996; Dworkin, 1980, 1981). Un feminismo para el 
que el "lugar" de la mujer en la especie es definido como el lugar de la "naturaleza" (Amorós, 1985). Y se declara la existencia de una esencia femenina, de un principio femenino, definido como la consagración de los llamados "valores femeninos" -a saber, dulzura, ternura y dedicación a los demás- y la denigración absoluta de los "valores masculinos". De esta forma, al interpretar la feminidad como algo inmutable, "natural", el feminismo cultural "asume que los hombres constituyen el enemigo por el hecho de ser hombres más que por el poder que un sistema patriarcal les ofrece" (Echols, 1983:441). En cualquier caso, cuando el feminismo cultural "elabora la idea de una superioridad de las mujeres y valora negativamente las cualidades atribuidas a los varones, adopta una perspectiva transhistórica porque la hace derivar de la maternidad y del amor maternal. Por añadidura, una visión de esta clase comporta a menudo la creencia en un matriarcado entendido, bien como lo que hemos perdido, bien como la utopía por venir, o ambas cosas a la vez" (Osborne, 2007:236).

Esta explicación subraya en exceso la rigidez del peso biológico (Harding, 1986, 1987), y hace derivar la "opresión de la mujer, no de la construcción del género, sino de la supresión de la esencia femenina, esencia que, por el contrario, se pretende perfilar por medio de un análisis radicalmente dicotómico del mundo. A un principio masculino se opone un principio femenino, muy claro en el ámbito de la sexualidad" (Osborne, 2007:218). Una sexualidad dicotomizada, ya que se tiene, por un lado, la sexualidad masculina, una sexualidad agresiva, irresponsable, orientada genitalmente y potencialmente letal. Por otro lado, la sexualidad femenina que se manifiesta de forma difusa, tierna, y se orienta a las relaciones interpersonales. $\mathrm{Y}$ es que ser naturaleza y poseer la capacidad de ser madres "comporta la posesión de las cualidades positivas, que inclinan en exclusiva a las mujeres a la salvación del planeta, pues para eso son moralmente superiores a los varones". De todo ello "se deduce la necesidad de una acentuación de las diferencias frente a las semejanzas entre los sexos, condenándose la heterosexualidad por su connivencia con el mundo masculino y revelándose el lesbianismo como la única alternativa de vida no susceptible de contaminación por el varón" (Osborne, 2007:217).

En definitiva, sobre la constatación del dimorfismo sexual se ha abierto una confrontación de perspectivas feministas antagónicas. Por un lado, el feminismo de la igualdad, de raíz ilustrada. Un feminismo que denuncia "las diferencias de género, porque entiende que lo masculino y lo femenino han sido el resultado de la construcción de la razón patriarcal. Propone, en consecuencia, la superación de los géneros en una sociedad no-patriarcal de individuos. El feminismo de la igualdad entiende que hoy sigue siendo posible la extensión de esta reivindicación ilustrada a las mujeres y propone, por tanto, como uno de sus objetivos prioritarios, desenmascarar cuánto de interés patriarcal hay en estas identidades de género -lo masculino y lo femenino-, en estos moldes genéricos que permiten perpetuar estereotipos, que no resultan ser nada favorables para las mujeres" (Posada, 
2007a:294). Por otro lado, el feminismo de la diferencia, entendida ésta como lo diferente, no como lo inferior, sino como "lo otro", como "lo no-idéntico". Un feminismo que se fundamenta en la crítica feroz al feminismo igualitario, al que tilda de reformista por demandar la equiparación de derechos entre hombres y mujeres. Para el feminismo de la diferencia "la dualidad de géneros no puede ser reclamada ni abolida, puesto que se trata de un orden dual que no es ni cultural ni biológico, sino que pertenece al orden de las cosas mismas -casi en un sentido existencial u ontológico" (Posada, 2007a:295). Como afirma Irigaray (1994:57-58), "lo natural es por lo menos dos: masculino y femenino. Todas las especulaciones sobre la superación de lo natural en lo universal olvidan que la naturaleza no es una (...) Así, también para estas dos partes del género humano, que son el hombre y la mujer. Sólo abusivamente son reducidas a uno. La razón muestra, en esta reducción, su impotencia o su inmadurez (...) El género humano, pues, no habría alcanzado la edad de la razón".

\section{LAS DOMINACIONES DE GÉNERO, CLASE SOCIAL, ETNIA, SEXUALIDAD...}

Dado que las diversas formas de estratificación social se interrelacionan en la vida de las mujeres, no se puede omitir en el análisis de la estratificación social el plano de las relaciones de género que están claramente cruzadas por las de clase, raza, etnia, edad, opción sexual. De hecho, es la posición de clase, la pertenencia étnica, la opción sexual de la mujer la que acaba estructurando el significado concreto que el género tiene para ella (Moore, 1999). En este sentido, para el feminismo "negro" o "multirracial" carece de sentido referirse a un sujeto femenino genérico, puesto que el plano de lo femenino es un ámbito internamente fragmentado por la clase, la raza, las características étnicas o la edad. En palabras de Butler (2001a:35), el género no es exhaustivo, "no es coherente o consistente en contextos históricos distintos"; su significado se construye invariablemente en relación con las modalidades raciales, de clase, étnicas, sexuales y regionales de cada situación (Guadarrama y Torres, 2007b). Por consiguiente, el género es una "relación entre sujetos socialmente constituidos en contextos específicos" (Butler, 2001a:43), de ahí que el feminismo postcolonial reclame análisis específicos contextualizados y diferenciados de las formas en que las mujeres se confirman como un grupo sociopolítico, histórico y cultural particular.

El planteamiento que efectúa Butler explica la posición del feminismo "negro". Así, para Hooks (1984), las feministas blancas nos han convertido en los "objetos" de su discurso privilegiado sobre la raza. Como "objetos", hemos permanecido desiguales, inferiores. Aunque puedan estar preocupadas sinceramente por el racismo, su metodología sugiere que no están libres del tipo de paternalismo endémico a la ideología de la supremacía blanca. Algunas de esas mujeres se colocan a sí mismas en la posición de "autoridades" que deben mediar 
la comunicación entre las mujeres blancas racistas y las mujeres negras enfadadas a quienes creen incapaces de elaborar discursos racionales. Según Amos y Parmar (1984), la percepción que las feministas blancas de clase media tienen de aquello de lo que necesitan liberarse tiene poca o ninguna relevancia para la experiencia del día a día de la mayoría de las mujeres negras. En palabras de Lorde (1984:117), "mientras las mujeres blancas ignoren su privilegio incorporado y definan a las mujeres únicamente en términos de su propia experiencia, las mujeres de color se convertirán en la otra, la extraña cuya experiencia y tradición es demasiado extraterrestre para poderse entender".

Reconocer la complicidad de las mujeres "privilegiadas" con la opresión o la perpetuación de las prácticas opresoras supone considerar las estructuras sociales y materiales de dominación, y asumir que las categorías de lo femenino y de lo masculino en el ámbito epistemológico "tienen importantes correlatos sociales. Porque sirven para clasificar a grupos sociales, otorgando a las personas determinados rasgos que les han de caracterizar, y para estructurar los espacios e instituciones sociales" (Pérez Orozco, 2007:32). De aquí que desde la denominada política de la localización (Rich, 1980a, 1986) se defina el género no como una construcción binaria y monolítica, sino como una marca de una posición de subordinación que está cualificada por otras variables de opresión. El género, inserto en una compleja red de relaciones de poder, no es el único determinante de la identidad de una persona y, al mismo tiempo, su forma concreta depende de esa red, y es que las variaciones que existen entre mujeres de diferentes clases, orientaciones sexuales y de diferentes orígenes étnicos son tan importantes para la posición social de la mujer como los elementos comunes inherentes al hecho de ser una mujer dentro de una sociedad determinada (Benería y Sen, 1983). Entonces, hay que situar a hombres y mujeres en múltiples sistemas de dominación (Parella, 2003), y explicar por qué hay mujeres y hombres que lo tienen todo y mujeres y hombres que no tienen nada (Baca y Thornton, 1994). De un modo más específico, hablar de dos géneros contribuye a esencializar las diferencias e ignorar la complejidad de las identidades sexuales y sociales posibles. De ahí que no se pueda olvidar relacionar el género con otras categorías que informan de las relaciones sociales y de las formas culturales (Beltrán y Maquieria, 2001; Juliano, 1994, 2004), y es que el gran error de la feminista de clase media es dar por supuesto que, con independencia del origen de clase, opción sexual y origen étnico, la experiencia del sexismo es la misma, como si en realidad existiera la "mujer genérica" (Parella, 2005a, 2005b), y como si en realidad existiera un dato a priori el sexo-, previo a su denominación, esto es, a una lectura interesada de los cuerpos que toma los datos físicos de éstos como causa de las prácticas sociales y no como efectos de procesos sociales que instituyen dispositivos disciplinarios que convierten a los propios cuerpos en algo relevante desde el punto de vista de la clasificación jerárquica de los sexos y/o géneros (Mora, 2005; Izquierdo, 2000, 2001; Butler, 2001a, 2001b). 
Ante esta jerarquización, no hay más alternativa que el cuestionamiento y la des-identificación en los mandatos y asignaciones de la "tecnología del género" (Lauretis, 1987). Como también hay que cuestionar los modos a través de los cuales las mujeres son construidas en los procesos étnicos y nacionales, tal y como es desarrollado en el feminismo postcolonial (Chakravorti Spivak, 1999a, 1999b; Femenías, 2000; Marre, 2001), definido como un conjunto amplio de trabajos que examinan la condición histórica de las mujeres en los países liberados de su dominación colonial después de la Segunda Guerra Mundial (Femenías, 2000). Un feminismo crítico de la lógica de dominio mundial que genera un centro (el Imperio) y una periferia (las colonias), vinculados entre sí "por un movimiento expansionista regulado desde dentro de las fronteras del Imperio por un discurso social que marca el lugar de la experiencia efectiva de la marginalidad, a la vez que permite su transformación en experiencia crítica" (Femenías, 2005:157).

El feminismo postcolonial representa una suerte de pérdida de la inocencia sobre las narrativas igualitarias modernas, su neutralidad y su potencial emancipatorio. Un feminismo que "regula las definiciones de qué somos, cómo somos, quiénes somos, desde el punto de vista de la cultura hegemónica. Así, el Pensamiento Postcolonial explora los niveles teóricos de las patologías sociales que giran en torno al antagonismo contingente como piso de una necesidad histórica. Sobre esa base, se elaboran estrategias de emancipación, planteando demandas culturales no sólo como un cambio de contenidos sino fundamentalmente como la resignificación simbólica de su inscripción histórica. Donde el multiculturalismo se ancla en el pensamiento postmoderno para intersectar las inscripciones narrativas del sujeto con la clase, el género, la opción sexual o la religión, el pensamiento postcolonial, por su parte, toma como punto de partida la lógica del dominio entendida a partir de la dialéctica binaria Uno-Otro, en términos de colonizadorcolonizado, y se mueve críticamente dentro de un marco moderno del poder" (Femenías, 2005:156-157).

En el multiculturalismo más radical, a las mujeres se las identifica en tanto que estarían dentro de una cultura específica, étnica o nacional, y al margen que ésta genere o no desigualdades y subordinaciones ilegítimas. Al respecto, Maquieira (2006a) afirma que las mujeres en los procesos étnicos y nacionales han sido construidas primero, como reproductoras biológicas de los miembros de la nación y las colectividades étnicas; segundo, como reproductoras de los límites de los grupos étnicos o nacionales; tercero, como participantes fundamentales en la reproducción ideológica de la colectividad y como transmisoras de su cultura; cuarto, como representación de las diferencias étnicas o nacionales, como un foco y símbolo en los discursos ideológicos utilizados en la construcción, reproducción y transformación de las categorías nacionales y étnicas, y quinto como participantes en las luchas nacionales, económicas, políticas y militares.

El énfasis en los roles reproductivos de las mujeres "se deriva de la importancia atribuida al origen o destino común en la construcción de gran parte de 
las identidades étnicas y nacionalistas. Por consiguiente, no es casual que las comunidades étnicas y los Estados preocupados con la pureza racial o étnica hayan reglamentado las relaciones sexuales de los miembros de las distintas colectividades y sobre todo el control de los cuerpos y la sexualidad de las mujeres para garantizar el crecimiento o freno de la cantidad y/o calidad de la población" (Maquieira, 2006a:74). Históricamente, las mujeres también han sido consideradas como depositarias, guardianas y transmisoras de la cultura. En este sentido, se les atribuye la reproducción cultural de la comunidad, del grupo étnico o de la nación. Como responsables del honor de la colectividad, "se prescribe para las mujeres espacios y comportamientos apropiados junto al uso de determinadas formas de vestir que marcan los límites simbólicos del grupo. A través de estos modos correctos en el vestir, los gestos y las conductas, las mujeres y las niñas se convierten en la personificación visible de los códigos culturales de su comunidad y ellas mismas en símbolos de lo auténticamente propio frente a lo ajeno. Muchas mujeres son torturadas o asesinadas por sus parientes cuando sus comportamientos son considerados una vergüenza o deshonra para sus familias y comunidades. Una situación que se agudiza cuando los Modelos de Estado y las identidades de las comunidades se vinculan a la religión, sacralizando así al patriarcado y retroalimentando las diversas formas de legitimación de la desigualdad" (Maquieira, 2006a:75).

De este modo mediante el esencialismo cultural se dota a la desigualdad de un carácter de inmutabilidad e irreversibilidad. Sin embargo, el feminismo postcolonial, en oposición "a los modelos de autoctonía racial y cultural, sostienen que en una era de constantes migraciones, de globalización de los mass-media y de continuo flujo transnacional de la información, la distinción colonial-postcolonial pura es imposible de sostener. La cultura de los colonizados -como ya había advertido Franz Fanon- irrevocablemente se altera en contacto con la de los colonizadores y viceversa. La narrativa postcolonial rompe la estructura dentro / fuera y con ella, las barreras que impiden resignificar los límites y producir lugares de experiencia nuevos. No hay posibilidad de espacios homogéneos" (Femenías, 2005:158-159). Maquieira (2006a:70), siguiendo a Juliano (1994), afirma que este esencialismo al defender la diferencia cultural la transforma paradójicamente en un importante elemento de segregación de los grupos subordinados y prácticas políticas sustentadas en la exclusión, ya que "al basarse en una conceptualización estática de la cultura esencializa la diferencia cultural haciéndola incompatible con otras formas culturales, y de este modo se convierte en el soporte de ideologías y prácticas que consideran peligrosa o contaminante la aceptación de individuos provenientes de orígenes étnicos diferentes, favoreciendo prácticas de segregación o de configuración de guetos marginales". De este modo, el esencialismo culturalista se convierte en la clave de la oposición nosotros / ellos y tiene también consecuencias importantes en el mantenimiento de la desigualdad de género. 
Las relaciones de género son "vistas a menudo como constitutivas de la esencia de las culturas y éstas como modos de vida indiscutibles que deben ser transmitidos de generación en generación" (Maquieira, 2006a:71). En este sentido, las tradiciones culturales y la (re)invención de las tradiciones son, a menudo, utilizadas como medios de legitimar la opresión de las mujeres. $Y$ en situaciones en las cuales tanto los varones individualmente como las colectividades étnicas o nacionales se sienten amenazadas/os, este fenómeno se puede intensificar. No es de extrañar, pues, "que las iniciativas de cambio de las mujeres se interpreten como una amenaza a la identidad cultural y a las tradiciones, ya que dado el papel asignado como reproductoras biológicas y simbólicas de la comunidad, se las convierte en objetos de control y culpables de la desintegración sociocultural. Ésta es la razón por la que la defensa de la diferencia cultural se convierte, en muchas ocasiones, en una nueva forma de legitimación de la desigualdad" (Maquieira, 2006a:75).

Amorós (2005a:222) afirma que el término multiculturalidad designa el hecho sociohistórico, incrementado en la era de la globalización, de la coexistencia de diversas culturas en los mismos ámbitos geográficos. Pues bien, el multiculturalismo radical pretende que cada cultura sea un bloque monolítico, "una totalidad autorreferida, homogénea y estática. Habría así una inconmensurabilidad radical entre los parámetros propios de las distintas culturas. No sería posible ni legítimo, de este modo, interpretar las diversas prácticas que se ejercen en el seno de cada cultura sino exclusivamente en función de los referentes de sentido de la cultura en cuestión. Dicho de otra forma, habría un monismo hermenéutico del significado, que sólo podría ser descifrado por quienes comparten el marco simbólico propio de cada totalidad cultural". Sin embargo, desde el punto de vista empírico, esta concepción de la cultura no se sostiene. Podemos tener alguna duda "sobre si se ajusta más o menos a la descripción de lo que habrían podido ser las culturas en un pasado más o menos remoto. Pero, desde luego, no es en absoluto adecuada para pensar las dinámicas culturales en el mundo de la globalización. Las culturas no son ni estáticas ni homogéneas, ni, mucho menos, totalidades autorreferidas. Tan importantes como los supuestos compartidos son en ellas los conflictos, las tensiones, los desajustes, los desgarramientos, los disensos y, desde luego, el fenómeno del cambio cultural". En la era de la globalización, se producen "constantemente hibridaciones, préstamos, apropiaciones selectivas de ciertos elementos de las culturas tanto hegemónicas como no hegemónicas junto con rechazos selectivos de otros. Nunca tiene lugar ni la total asimilación ni la total destrucción de una cultura por otra: también las culturas hegemónicas son siempre en alguna medida penetradas por las que no lo son. Los seres humanos no se limitan a ser agentes de la reproducción sociocultural: lo son también de la revisión de los códigos culturales" (Amorós, 2005a:224). La revisión de los códigos culturales significa, además, una vía de emancipación, y para las mujeres emanciparse significa emanciparse con respecto a su situación de subordinación, 
que pasa necesariamente "por un proceso en el que pongan en cuestión la diferencia genérica que les ha sido asignada como una construcción -política, cultural, simbólica- a la que no quieren estar sujetas y de la cual, en esa misma medida, se des-identifican" (Amorós, 1997:19). Esto supone, para Braidotti (2004), defender la idea de que cada persona tiene múltiples identidades parciales entre las que puede viajar, por propia voluntad o por imposición social, y que esa posibilidad misma de viajar hace que los sujetos no tengan una identidad fija, sino situada en el tiempo y el espacio. El movimiento de atravesar fronteras de la identidad es un proceso del cual participa activamente la propia persona, en una "labor autoconstructora", por lo que no puede entenderse como mera víctima de un determinismo social o biológico.

Braidotti (1994) habla del sujeto nómada como alternativa a "la mujer" o "las mujeres". La condición nómada "es una nueva figuración de la subjetividad de un modo multidiferenciado no jerárquico. Nómadas porque atraviesan estereotipos y fronteras de la identidad, por obligación o elección, más aún en los actuales tiempos de globalización y migraciones. La identidad se entiende como un juego de fronteras móviles, productoras de significados, poderes y estructuras; no simplemente viajamos atravesando fronteras, sino que somos fronteras. La pureza categorial que se ceñía a los márgenes impuestos por los sistemas de poder (capitalismo, patriarcado, racismo...) dando como resultado identidades fijas y coherentes (la clase, el género, la etnia...) se ha desterrado" (Pérez Orozco, 2007:25-26). Otra nueva figuración es el ubicuo cyborg de Haraway (1995). El cyborg se ha convertido en un icono de la idea de que se han desdibujado los límites entre lo biológico y lo cultural, así como entre el ser humano y la máquina. Estas dicotomías solían posicionar a las mujeres en lo natural y lo diferente y servían para sustentar el orden genérico previamente establecido. Al romper, como lo hacen estas nuevas tecnologías del cuerpo, el vínculo entre feminidad y maternidad, se alteran las categorías de cuerpo, sexo, género y sexualidad. Esto redunda en una liberación para las mujeres, que han estado cautivas de la biología, por lo que el cyborg constituye la figuración de las nuevas subjetividades políticas, y que Haraway caracteriza por contraposición a tres referentes polémicos: la subjetividad unitaria, la totalidad orgánica y la filosofía teleológica de la historia. Sin embargo, frente a la figuración cyborg, está el fantasma de la ingeniería genética y de la clonación, que priva a las mujeres de cualquier control sobre la reproducción. En esta visión apocalíptica, "la tecnociencia está profundamente arraigada en el proyecto masculino de dominación y de control de las mujeres y de la naturaleza. El tropo clásico de la ciencia-ficción, Frankenstein y su monstruo, se invoca como el lado oscuro de cyborg -vida artificial fuera de control. Crece el temor acerca de la manera en que pueda utilizarse el conocimiento del genoma para intervenir en la naturaleza y rediseñarla, tanto si se trata de alimentos genéticamente modificados como de animales clonados o de seres humanos de una raza perfeccionada. La propia vida (humana, vegetal y animal) corre el riesgo de 
que se biomedicalice y se mercantilice. La ingeniería genética y reproductiva se entiende por lo tanto como un intento más de usurpar a las mujeres la autodeterminación sobre su cuerpo" (Wajcman, 2006:15).

\section{CONCLUSIÓN}

Decíamos en la introducción que la división del trabajo es división sexual; observable no únicamente en el vínculo entre la feminización de la pobreza y el empeoramiento de las condiciones de trabajo, sino también en el contenido y las condiciones de trabajo hoy. Condiciones impuestas tras violentas reestructuraciones y que no son más que la extensión tendencial de las características del trabajo, tanto asalariado como no asalariado, estructural e históricamente asignado a las mujeres, al trabajo en sentido genérico. El orden sexual da cuenta de cómo el orden social está profundamente generizado, y que la actividad económica se haya interpretado a través de categorías masculinas mediante valores androcéntricos. En sentido estricto, la naturaleza del trabajo tiene una clara base en el sexo, dado que no es la división del trabajo lo que explica la subordinación de las mujeres, sino que es la desigualdad entre hombres y mujeres lo que se incorpora como factor estructurante en las relaciones de producción y en la división del trabajo. Desde este punto de vista, son las desigualdades de género las que determinan la posición que hombres y mujeres ocupan en la producción de la existencia (Gardiner, 1997; Borderías et al., 1994; Ferber y Nelson, 1993; Lagrave, 1993; Bourdieu, 2000), dado que todavía hoy el proceso de socialización está organizado jerárquicamente entorno a los géneros, el que produce y reproduce la vida humana, y el que produce y administra los medios que permiten la ampliación de la vida humana. Esta organización forma parte de un sistema de dominación masculina, y sin esta dominación, el patriarcado "quedaría desenmascarado como una dominación arbitraria y acabaría siendo derrocado por la rebelión de la mitad del cielo mantenida bajo sometimiento a lo largo de la historia" (Castells, 1998:158). Bajo esta dominación en la actualidad se observa que la posición económica de las mujeres se viene deteriorando, por su situación en el mercado laboral, en el trabajo doméstico y por el escaso acceso a la protección contributiva. Además, el estudio de la pobreza y de la exclusión social de las mujeres en España carece de una trayectoria consolidada, existiendo referencias a la denominada feminización de la pobreza (Fernández, 1993; López Larrea, 1989; Martínez-Román, 1997; Regidor et al., 1994), pero existe un déficit generalizado desde una perspectiva de género. Déficit inexplicable si asumimos que las diferencias de género configuran la vida de hombres y mujeres en aspectos fundamentales (por ejemplo, la distribución de ingresos, riqueza y tiempos), por lo que hay que transformar las relaciones de género si se quiere mejorar la situación de las mujeres; relaciones que son claves para comprender la situación social y económica de las mismas, ya que al ser éstas las encargadas de realizar el trabajo 
doméstico, cuentan con un doble problema. Por un lado, la dedicación al cuidado de personas mayores, enfermos o niños obliga a reducir o suprimir toda actividad remunerada. Ello supone una pérdida de la independencia económica al mismo tiempo que se empobrece su red de relaciones sociales. Por otro lado, el hecho de que las mujeres sean dependientes del ingreso de sus cónyuges las sitúa automáticamente en una situación muy vulnerable, cuando cualquier acontecimiento vital afecte al cónyuge. Éste es el caso, señala La Parra (2001), de los hogares monoparentales o de las viudas, que se encuentran entre los grupos más vulnerables a la pobreza en nuestra sociedad. Esta situación se debe a que en el momento en que "el trabajo se convertía en un valor central que afianzaba socialmente la existencia del individuo y se situaba así en el corazón de la ciudadanía libre e igualitaria, las mujeres se veían excluidas del trabajo, de la individualidad social y de la ciudadanía" (Daune-Richard, 2007:242). Esta exclusión obedecía a que las mujeres fueron definidas como "dependientes" de los vínculos familiares y domésticos. Al convertirse "la relación con el trabajo y el empleo en vínculo individual en la nueva organización social (puesto que dependía del contrato), ellas no podían integrarse plenamente en ella. Así, durante mucho tiempo, el vínculo familiar será el que defina su vínculo con el empleo: o contribuyen a la producción de la empresa familiar, en la medida en que el marido es al mismo tiempo cabeza de familia y empresario, o acceden al mercado de trabajo asalariado, aunque sea bajo control del marido" (Daune-Richard, 2007:243).

\section{BIBLIOGRAFÍA}

ALLO, F. y HARCOURT, W. (1997): "From South to the North; evolving perspectives on gender and poverty", Gender and development, vol. 5, n. 3, pp. 9-12.

AMORÓS, C. (1985): Hacia una crítica de la razón patriarcal, Barcelona, Anthropos.

- (1997): Tiempo de feminismo. Sobre feminismo, proyecto ilustrado y postmodernidad, Madrid, Cátedra.

- (2005a): "Feminismo y multiculturalismo", en Amorós, C. y De Miguel, A. (eds.): Teoría feminista: de la Ilustración a la globalización. Vol. 3. De los debates sobre el género al multiculturalismo, Madrid, Minerva.

- (2006): La gran diferencia y sus pequeñas consecuencias... para las luchas de las mujeres, Madrid, Cátedra. Col. Feminismos.

AMORÓS, C. y DE MIGUEL, A. (eds.): "Teoría feminista y movimientos feministas", en Amorós, C. y De Miguel, A. (eds.) (2007c): Teoría feminista: de la Ilustración a la globalización. Vol. 1. De la Ilustración al segundo sexo, Madrid, Minerva.

AMOS, V. y PARMAR, P. (1984): "Challenging Imperial Feminism", en Bhavnani, K., (ed.) (2001a): Feminism and Race, Oxford, Oxford University Press

ANDERSEN, J. y LARSEN, J. (1998): "Gender, poverty and empowerment", Critical social policy, 55, pp. 241-258.

BACA, M. y THORNTON, B. (1994): "Difference and Domination", en Baca, M. y Thornton, B. (ed.): Women of Color in U.S. Society. Philadelphia, Temple University Press. 
BARRY, K. (1987): Esclavitud sexual de la mujer, Barcelona, La Sal.

- (1994): “Teoría del feminismo radical: política de explotación sexual”, en Amorós, C. (coord.): Historia de la teoría feminista. Madrid, Dirección General de la Mujer de la Comunidad de Madrid, Instituto de Investigaciones Feministas de la Universidad Complutense.

- (2007): "Teoría del feminismo radical: política de la explotación sexual”, en Amorós, C. y De Miguel, A. (eds.): Teoría feminista: de la Ilustración a la globalización. Vol. 2. Del feminismo liberal a la posmodernidad, Madrid, Minerva.

BELTRÁN, E. y MAQUIEIRA, V. (ed.) (2001): Feminismos. Debates teóricos y contemporáneos, Madrid, Alianza Editorial.

BENERÍA, L. (1992): “Accounting for women Work: the Progress of Two Decades", World Development, vol. 20 (1), pp. 63-95.

BENERÍA, J. y SEN, G. (1983): "Desigualdades de clases y de género y el rol de la mujer en el desarrollo económico: implicaciones teóricas y prácticas”, Mientras Tanto, vol. 15, núm. 5, pp. 91-113.

BENHABIB, S. (1990): "El Otro generalizado y el otro concreto", en Benhabib, S. y Cornell, D. (eds.): Teoría feminista y teoría crítica, Valencia, Edicions Alfons el Magnànim.

BORDERÍAS, C.; CARRASCO, C. y ALEMANY, C. (1994): Las mujeres y el trabajo. Rupturas conceptuales, Madrid, Icaria-Fuhem

BOSERUP, E. (1993): La mujer y el desarrollo económico, Madrid, Minerva Ediciones.

BOURDIEU, P. (2000): La dominación masculina, Barcelona, Anagrama.

BRAIDOTTI, R. (1994): Sujetos nómadas, Buenos Aires, Paidós.

- (2004): Feminismo, diferencia sexual y subjetividad nómada, Barcelona, Gedisa.

BRULLET, C. (2004): "La maternidad en occidente y sus condiciones de posibilidad en el siglo XXI", en De La Concha, A. y Osborne, R. (coords.): Las mujeres y los niños primero. Discursos de la maternidad, Barcelona, Icaria.

BUTLER, J. (1990): "Variaciones sobre sexo y género. Beauvori, Wittig y Foucault", en Benhabib, S. y Cornell, D. (comp.) : Teoría Feminista y teoría crítica. València, Edicions Alfons el Magnànim, Institució Valenciana dEstudis i Investigació.

- (2001a): El género en disputa. México, D. F., Paidós, Género y Sociedad.

- (2001b): Mecanismos psíquicos del poder. Madrid, Cátedra, Feminismos, 68.

CARRASCO, C.; ALABART, A.; COCO, A.; DOMÍNGUEZ, M.; MARTÍNEZ, A.; MAYORDOMO, M.; RECIO, A. y SERRANO, M. (2003): Tiempos, trabajos y flexibilidad: una cuestión de género, Madrid, Instituto de la Mujer.

CASTAÑO, C.; IGLESIAS, C.; MAÑAS, E. y SÁNCHEZ-HERRERO, M. (1999): Diferencia o discriminación: La situación de las mujeres españolas en el mercado de trabajo y el impacto de las nuevas tecnologías de la información. Madrid, CES.

CHAKRAVORTI SPIVAK, G. (1999a): “PPuede el sujeto subalterno hablar?”, Orbius Tertius, 6, pp. 175-235.

- (1999b): A critique of Postcolonial Reason, Londres, Harvard University Press.

DAUNE-RICHARD, A. M. (2007): "Las mujeres y la sociedad salarial: una investigación a partir de los casos de Francia, Reino Unido y Suecia”, en Prieto, C. (ed.): Trabajo, género y tiempo social, Barcelona, Hacer.

DWORKING, A. (1980): "Why So-Called Radical Men Love and need Pornography", en Lederer, L. (ed.): Take Back the Night, New York, William Morrow.

DWORKING, A. (1981): Pornography, New York, Perigee Books. 
ECHOLS, A. (1983): “The New Feminism of Yin and Yang”, a Snitow, A., Stansell, C., y Thompson, Sh. (eds.): Powers of Desire. The Politics of Sexuality, New York, Monthly Review Press.

FEMENÍAS, M. L. (2000): Sobre sujeto y género: lecturas feministas de Beauvoir a Butler, Buenos Aires, Catálogos.

- (2005): "El feminismo postcolonial y sus límites", en Amorós, C. y De Miguel, A. (eds.) Teoría feminista: de la Ilustración a la globalización. Vol. 3. De los debates sobre el género al multiculturalismo, Madrid, Minerva.

FERBER, M. y NELSON, J. (eds.) (1993): Más allá del hombre económico, Madrid, Cátedra, la Universidad de Valencia y el Instituto de la Mujer.

FERNÁNDEZ, B. (1993): "La feminización de la pobreza", Mujeres en Acción, núm. 4 extraordinario, pp. 14-23.

FOLGUERA, P. (2006a): "La equidad de género en el marco internacional y europeo", en Maquieira, V. (ed.), Mujeres, globalización y derechos humanos, Madrid, Cátedra.

FRIEDAN, B. (1974): La mística de la feminidad, Madrid, Júcar.

GARDINER, J. (1997): Gender, Care and Economics, London, MacMillan Press Ltd.

GREER, G. (1985): Sexo y destino, Barcelona, Plaza y Janés.

- (2000): La mujer completa. Barcelona, Kairós.

GUADARRAMA, R. y TORRES, J. L. (coords.) (2007b): Los significados del trabajo femenino en el mundo global. Estereotipos, transacciones y rupturas, Barcelona, Anthropos.

HARAWAY, D. (1995): Ciencia, cyborgs y mujeres. La reinvención de la naturaleza, Madrid, Cátedra. Col. Feminismos.

HARDING, S. (1986): The Science Question in Feminism, Cornell University Press, New York, Ithaca.

- (ed.) (1987): Feminism and Methodology: Social Science Issues, Bloomington, Indiana University Press.

HARTMANN, H. (1981): “The Unhappy Marriage of Marxism and Feminism: Towards a More Progressive Union”, en Sargent, L. (comp.): Women and Revolution, Boston, South End Press.

- (1994): “Capitalismo, patriarcado y segregación de los empleos por sexo”, en Borderías, C. et al. (eds.): Las mujeres y el trabajo, Madrid, Icaria- Fuhem.

HOOCKS, B. (1984): "Black Women: Shaping Feminist Theory" en Bhavnani (ed.). Feminism and Race, New York, Oxford University Press.

IRIGARAY, L. (1994): Amo a ti, Barcelona, Icaria.

IZQUIERDO, M. J. (2000): Cuando los amores matan, Madrid, Libertarias. Saber vivir.

- (2001): Sin vuelta de hoja. Sexismo: poder, placer y trabajo, Barcelona, Bellaterra, La Biblioteca del Ciudadano.

JEFFREYS, S. (1996): La herejía lesbiana. Una perspectiva feminista de la revolución sexual lesbiana, Madrid, Cátedra.

JÓNASDÓTTIR, A. (1993): El poder del amor. Le importa el sexo a la democracia? Valencia, Cátedra, Col. Feminismos.

JULIANO, D. (1994): “La construcción de la diferencia: los latinoamericanos", Papers, 43: 23-32.

- (2004): Excluidos y marginales, Madrid, Cátedra.

KABEER, N. (1997): “Tactics and trade-off: revisiting the links between gender and poverty", Institute of Development Studies Bulletin, 28. 
- (1998): Realidades trastocadas. Las jerarquías de género en el pensamiento del desarrollo, México, Paidós.

LAGARDE, M. (1996): Género y feminismo. Desarrollo humano y democracia. Madrid, Horas y Horas.

LAGRAVE, R. M. (1993): “Una emancipación bajo tutela. Educación y trabajo de las mujeres en el siglo XX”, en Duby, G. y Perrot, M., Historias de las mujeres, Madrid, Taurus.

LA PARRA, D. (2001): "Vida familiar y empobrecimiento", en Tortosa, J. M. (coord.): Pobreza y perspectiva de género, Barcelona, Icaria.

LAURETIS, T. de (1987): Technologies of Gender. Essay in Theory, Films and Fiction" Bloomington, Indiana University Press.

- (2000): Diferencias, Madrid, Horas y Horas.

LÓPEZ LARREA, M. F. (1989): "La feminización de la pobreza", Cuadernos de acción social, núm. 14, pp. 51-55.

LORDE, A. (1984): "The Masters Tools Will Never Dismantle the Masters House", en Lorde, A. (ed.): Sister Outsider: Essays and Speeches, Santa Cruz, The Crossing Press.

MAQUIEIRA, V. (2006a): "Mujeres, globalización y derechos humanos", en Maquieira, V. (ed.): Mujeres, globalización y derechos humanos, Madrid, Cátedra.

- (2006b). Mujeres, globalización y derechos humanos. Madrid: Cátedra.

MARCOUX, A. (1998): "The Feminization of Poverty: Claims, Facts, and Data Needs", Population and Development Review, vol. 24, $\mathrm{n}^{\circ}$. 1, pp. 131-139.

MARRE, D. (2001): "La continuidad de la exclusión en el proceso de construcción de la nación: ediciones y (re)ediciones, en Nahs, M., y Marre, D., Multiculturalismos y género, Barcelona, Bellaterra.

MARTÍNEZ ROMÁN, M. A. (1997): "Política social, pobreza y exclusión social”, en Alemán, C. y Garcés, J.: Política social, Madrid, McGraw Hill.

MARTÍNEZ, M. A. (2001): “Género, pobreza y exclusión social: diferentes conceptualizaciones y políticas públicas”, en Tortosa, J. M. (coord.): Pobreza y perspectiva de género, Barcelona, Icaria.

MARUANI, M. (2002): Trabajo y el empleo de las mujeres, Madrid, Editorial Fundamentos.

- (2007): “Tiempo, trabajo y género", en Prieto, C. (ed.): Trabajo, género y tiempo social, Barcelona, Hacer.

MARUANI, M., ROGERAT, C. y TORNS, T. (dirs.) (2000): Las nuevas fronteras de la desigualdad. Hombres y mujeres en el mercado de trabajo, Barcelona, Icaria.

MILLET, K. (1995): Política sexual, Madrid, Ed. Cátedra. Col. Feminismos.

MOLINA, C. (1994a): "El feminismo socialista contemporáneo en el ámbito anglosajón", en Amorós, C. (coord.) Historia de la Teoría feminista, Madrid, Instituto de Investigaciones Feministas. Universidad Complutense de Madrid.

- (1994b): Dialéctica Feminista de la Ilustración, Barcelona, Anthropos.

- (2000): "Debates sobre el género", en Amorós, C. (ed) Feminismo y Filosofía, Madrid, Síntesis.

- (2007): "El feminismo socialista estadounidense desde la Nueva Izquierda. Las teorías del sistema dual (Capitalismo + Patriarcado)", en Amorós, C., y De Miguel, A. (eds.) Teoría feminista: de la Ilustración a la globalización, vol. 2. Del feminismo liberal a la posmodernidad, Madrid, Minerva.

MOORE, H. L. (1999): Antropología y feminismo, Madrid, Cátedra. 
MORA, E. (2005): "Patriarcado, capitalismo y clases sociales", en Giró, J. (ed.) El género quebrantado. Sobre la violencia, la libertad y los derechos de la mujer en el nuevo milenio, Madrid, Catarata.

MURARO, L. (1992): "Sobre la autoridad femenina”, en Birulés, F. (comp.) Filosofía y género. Identidades femeninas, Pamplona, Pamiela.

- (1994): El orden simbólico de la madre, Madrid, Horas y Horas.

OLIVA PORTOLES, A. (2007): "La teoría de las mujeres como clase social: Christine Delphy y Lidia Falcón”, en Amorós, C. y De Miguel, A. (eds.): Teoría feminista: de la Ilustración a la globalización. Del feminismo liberal a la posmodernidad, Madrid, Minerva Ediciones.

OSBORNE, R. (2007): "Debates en torno al feminismo cultural", en Amorós, C. y De Miguel, A. (eds.): Teoría feminista: de la Ilustración a la globalización, vol. 2. Del feminismo liberal a la posmodernidad, Madrid, Minerva.

PARELLA, S. (2003): Mujer, inmigrante, trabajadora: la triple discriminación, Barcelona, Anthropos.

- (2005a): "La maternidad a distancia de las empleadas domésticas latinoamericanas en España. La vulneración del derecho a la vida familiar en el contexto de la internacionalización de la reproducción", en Giró, J. (ed.): El género quebrantado. Sobre la violencia, la libertad y los derechos de la mujer en el nuevo milenio, Madrid, Catarata.

- (2005b): "Segregación laboral y vulnerabilidad social de la mujer inmigrante a partir de la interacción entre clase social, género y etnia", en Solé, C. y Flaquer, LL. (eds.): El uso de las políticas sociales por las mujeres inmigrantes, Madrid, Instituto de la Mujer.

PATEMAN, C. (1995): El contrato sexual, Barcelona, Anthropos.

PÉREZ OROZCO, A. (2007): Perspectivas feministas en torno a la economía: el caso de los cuidados, Madrid, Consejo Económico y Social.

PERONA, A. (2007): "El feminismo liberal estadounidense de posguerra: Betty Friedan y la refundación del feminismo liberal", en Amorós, C. y De Miguel, A. (eds.): Teoría feminista: de la Ilustración a la globalización, vol. 2. Del feminismo liberal a la posmodernidad, Madrid, Minerva.

PICCHIO, A. (1992): Social Reproduction: the Political Economy of the Labour Market, Cambridge, Cambridge University Press.

- (1999): "Visibilidad analítica y política del trabajo de reproducción social", en Carrasco, C., "Mujeres en economía. Nuevas perspectivas para viejos y nuevos problemas", Barcelona, Icaria, Antrazyt, núm. 147.

PNUD (1995): Informe sobre Desarrollo Humano 1994, México, Fondo de Cultura Económica.

POSADA, L. (1998): Sexo y esencia. De esencialismos encubiertos y esencialismos heredados, Madrid, Horas y Horas.

- (2000): "De discursos estéticos, sustituciones categoriales y otras operaciones simbólicas: en torno a la filosofía del feminismo de la diferencia", en Amorós, C. (ed.) Feminismo y Filosofía, Madrid, Síntesis.

- (2001): "Las hijas deben ser siempre sumisas (Rousseau). Discurso patriarcal y violencia contra las mujeres: reflexiones desde la teoría feminista", en Bernárdez, A. (ed.) Violencia de género y sociedad: una cuestión de poder. Madrid, Instituto de Investigaciones Feministas, Ayuntamiento de Madrid. 
- (2007a): "La diferencia sexual como diferencia esencial: sobre Luce Irigaray", en Amorós, C. y De Miguel, A. (eds.): Teoría feminista: de la Ilustración a la globalización. Del feminismo liberal a la posmodernidad, Madrid, Minerva Ediciones.

- (2007b): "El pensamiento de la diferencia sexual: El feminismo italiano. Luisa Murazo" y "El orden simbólico de la madre", en Amorós, C. y De Miguel, A. (eds.) Teoría feminista: de la Ilustración a la globalización. Del feminismo liberal a la posmodernidad, Madrid, Minerva Ediciones.

PULEO, A. (2007): "Lo personal es político: el surgimiento del feminismo radical", en Amorós, C. y De Miguel, A. (eds.): Teoría feminista: de la Ilustración a la globalización, vol. 2. Del feminismo liberal a la posmodernidad, Madrid, Minerva.

RICH, A. (1980a): "Compulsory Heterosexuality and lesbian existence", en Abelove et al. (eds.): The Lesbian and Gays Studies Readers, NewYork, Routledge.

- (1986): Blood, Bread and Poetry: Selected Prose 1979-1985, Londres, Virago.

SCOTT, S. W. (1984): Gender and the Politics of History, New York, Columbia University.

TORTOSA, J. M. (1993): La pobreza capitalista, Madrid, Tecnos.

- (coord.), (2001): Pobreza y perspectiva de género, Barcelona, Icaria.

WAJCMAN, J. (2006): El tecno feminismo, Madrid, Ediciones Cátedra.

WITTIG, M. (1981): “One Is Not Born a Woman”, en Feminist Issues, vol. 1, núm. 2.

\section{Breve currículo:}

\section{Ignasi Brunet Icart}

Catedrático de Sociología de la Universitat Rovira i Virgili de Tarragona. Director del Programa de Doctorado "Organización Industrial: Empresas y Mercados", distinguido con la Mención de Calidad por el Ministerio de Educación y Ciencia. Las actuales líneas de investigación en que trabaja son: organización industrial, pymes y modelos de organización, mercados de trabajo y empleabilidad, estrategia empresarial, género y creación de empresas, género y pobreza. Sus últimos libros publicados son: Brunet, I. y Belzunegui, A., Teorías sobre la empresa, (2005); Brunet, I. y Alarcón, A., ¿Quién crea empresas? Redes y empresarialidad, (2005); Brunet, I. y Böcker, R., Desarrollo, industria y empresa, (2007); Brunet, I. y Vidal, A., El gobierno del factor humano, (2008). 
